\title{
Suplementação de ácidos graxos ômega 3 em atletas de competição: impacto nos mediadores bioquímicos relacionados com o metabolismo lipídico*
}

Priscila de Mattos Machado Andrade, Beatriz Gonçalves Ribeiro e Maria das Graças Tavares do Carmo

\section{RESUMO}

Objetivo: Avaliar o efeito da suplementação dos ácidos graxos ômega 3 em atletas de natação sobre indicadores bioquímicos. Métodos: Nadadores de elite $(n=14)$ do sexo masculino foram avaliados em estudo randomizado, controlado por placebo pelo período de seis semanas (45 dias). O grupo placebo (GP) recebeu óleo mineral $(n=6)$ e o grupo suplementado $(n=8)$, óleo de peixe (GOP) contendo, no total, 950mg de ácido eicosapentaenóico e $500 \mathrm{mg}$ de ácido docosapentaenóico. Amostras de sangue foram coletadas imediatamente antes (T0), aos 15 (T15), aos 30 (T30) e aos 45 (T45) dias de suplementação para análise da composição dos ácidos graxos por cromatografia gasosa e para quantificação das lipoproteínas plasmáticas através de kits comerciais específicos. Resultados: Os resultados revelaram um desajuste na dieta dos atletas considerando a ingestão $\mathrm{g} / \mathrm{kg}$ de massa corporal dos macronutrientes. A análise do questionário de freqüência de consumo mostrou que os atletas não ingeriram regularmente fontes alimentares de ômega 3 e que o consumo de peixes, em $85 \%$ da amostra, era inferior ou igual a uma vez na semana. O perfil de ácidos graxos plasmáticos evidenciou aumento dos ácidos graxos poliinsaturados ômega $3(P<0,05)$ e redução do ácido araquidônico no grupo suplementado $(P<0,05)$. A suplementação com óleo de peixe ocasionou efeito hipocolesterolêmico, com redução nos teores sanguíneos de VLDL, LDL e colesterol total. Os valores de HDL não apresentaram diferenças significativas entre os grupos em nenhum momento estudado $(P>0,05)$. Conclusão: $A$ suplementação de ácidos graxos $\mathrm{N}-3$ em atletas nadadores altera os indicadores bioquímicos do metabolismo lipídico, influenciando na redução das lipoproteínas plasmáticas, ricas em colesterol e na prevenção de doenças cardiovasculares.

\section{ABSTRACT \\ Omega 3 fatty acids-supplementation to competition athletes: impact on the biochemical indicators related to the lipid metabolism}

Purpose: To assess the effects of omega 3 fatty acid supplementation to swimmers on biochemical indicators. Methods: Male elite swimmers $(n=14)$ were assessed in a placebo-controlled randomized study over a 6-week (45-day) experimental period. The placebo group $(G P)$ received mineral oil $(n=6)$ and the supple-

* Instituto de Nutrição J osué de Castro da Universidade Federal do Rio de J aneiro, Rio de J aneiro.

Dedicamos este artigo ao Dr. Costa Rosa (GG) in memoriam pelo seu grande incentivo e amizade durante toda a realização do trabalho.

Recebido em 9/2/06. Versão final recebida em 8/5/06. Aceito em 6/6/06.

Endereço para correspondência: Dra. Maria das Graças Tavares do Carmo, Universidade Federal do Rio de J aneiro, Centro de Ciências da Saúde, Instituto de Nutrição, Bloco J - 2o andar - 21941-590 - Rio de J aneiro, RJ , Brasil. Fax: 5521 280-8343. E-mail: tcarmo@editema.com.br

\begin{abstract}
Palavras-chave: Atletas nadadores. Suplementação com óleo de peixe. Lipoproteínas.

Keywords: $\quad$ Swimmers. Fish oil supplementation. Lipoproteins.

Palabras-clave: Atletas nadadores. Suplementación con aceite de pescado. Lipoproteínas.
\end{abstract}

mented group $(G O P)$ received fish oil $(n=8)$ containing a total of $950 \mathrm{mg}$ of eicosapentaenoic acid and $500 \mathrm{mg}$ of docosapentaenoic acid. Immediately before starting the supplementation (TO), as well as 15 (T15), 30 (T30) and 45 (T45) days after that point, blood samples were collected and analyzed by gas chromatography for fatty acids composition, and by specific commercial kits for plasmatic lipoproteins. Results: The results showed that the diets of the swimmers were unbalanced regarding the macronutrient ingestion/body weight ratio $(\mathrm{g} / \mathrm{kg})$. The analysis of the consumption frequency questionnaire showed that (1) the swimmers have not regularly ingested omega 3 dietary sources and (2) the fish consumption was below once a week for $85 \%$ of the sample. The plasmatic fatty acids profile presented an increase in omega 3 polyunsaturated fatty acids $(p<0.05)$ and decrease in arachidonic fatty acid in the supplemented group ( $p<0.05$ ). The fish oil supplementation led to a hypocholesterolemic effect, with a decrease in VLDL, LDL and total cholesterol blood levels. The HDL levels presented no significant differences between the groups in any moment of the study ( $p>0.05)$. Conclusion: $N-3$ fatty acids supplementation to swimmers alters the biochemical indicators of the lipid metabolism, with an influence in the decrease of the cholesterol-rich plasmatic lipoproteins, so preventing cardiovascular diseases.

\section{RESUMEN}

Adición de ácidos grasos omega 3 en atletas de competición: impacto en los mediadores bioquímicos relacionados con el metabolismo lipídico

Objetivo: Evaluar el efecto suplementar de los ácidos grasos omega 3 en atletas de natación sobre indicadores bioquímicos. Métodos: Nadadores de elite $(n=14)$ del sexo masculino fueron evaluados en estudio aleatorio, controlado por placebo por un período de 6 semanas (45 días). El grupo placebo (GP) recibió aceite mineral $(n=6)$ y el grupo suplementado $(n=8)$ recibió aceite de pescado (GOP) conteniendo en total $950 \mathrm{mg}$ de ácido eicosapentaenóico y 500 mg de ácido docosapentaenóico. Muestras de sangre fueron colectadas inmediatamente antes (TO), a los quince (T15), a los treinta (T30) y a los cuarenta y cinco (T45) días de suplementación para análisis de la composición de los ácidos grasos por cromatografía gaseosa y para cuantificación de las lipoproteínas plasmáticas a través de kits comerciales específicos. Resultados: Los resultados revelaron un desajuste en la dieta de los atletas considerando la ingestión $\mathrm{g} / \mathrm{kg}$ de masa corporal de los macro nutrientes. El análisis del cuestionario de frecuencia de 
consumo mostró que los atletas no ingirieron regularmente fuentes alimentares de omega 3 y que el consumo de pescado, en $85 \%$ de la muestra, era inferior o igual a 1 vez por semana. El perfil de ácidos grasos plasmáticos mostró un aumento de los ácidos grasos poliinsaturados omega $3(P<0,05)$ y reducción del ácido araquidónico en el grupo suplementado $(P<0,05)$. La suplementación con aceite de pescado ocasionó efecto hipocolesterolémico, con reducción en los grados sanguíneos de VLDL, LDL y colesterol total. Los valores de HDL no presentaron diferencias significativas entre los grupos en ningún momento estudiado $(P>$ 0,05). Conclusión: La suplementación de ácidos grasos N-3 en atletas de natación altera los indicadores bioquímicos del metabolismo lipídico, influyendo en la reducción de las lipoproteínas plasmáticas, ricas en colesterol y en la prevención de enfermedades cardiovasculares.

\section{INTRODUÇÃO}

Nos últimos anos tem havido grande interesse, por parte da comunidade científica, pelos ácidos graxos poliinsaturados ômega 3, principalmente EPA (eicosapentaenoic acid) e DHA (docosahexaenoic acid), encontrados em peixes e óleos de peixe. A base desse interesse, pela ingestão dietética de EPA e DHA, vem de estudos populacionais e epidemiológicos, os quais mostraram que o consumo de peixe está associado a diminuição dos coeficientes de morbimortalidade pelas doenças cardiovasculares ${ }^{(1)}$. Os ácidos graxos poliinsaturados ômega 3 (AGPI N-3) necessitam estar no organismo numa proporção adequada para apresentar esses efeitos benéficos. A relação dietética de AGPI N-6/N-3 deve estar entre 3:1 a 5:1(2). As dietas ocidentais possuem elevado teor de AGPI N-6, estabelecendo a relação entre eles de 10 a $25: 1^{(3-4)}$. Nesse contexto, atualmente recomenda-se maior consumo de AGPI N-3 devido à baixa ingestão desses ácidos graxos pela população. De fato, observa-se decréscimo no consumo de peixes, principal fonte de AGPI N-3, e maior produção de rações para animais como frango e peixes de cativeiro, ricas em AGPI N-6(2). Em resumo, a agricultura modema promoveu nos últimos anos redução do teor de AGPI N-3 em vários alimentos como ovos, hortaliças e cames ${ }^{(2)}$.

Os efeitos hipolipidêmicos, antitrombóticos e antiinflamatórios dos AGPI N-3 têm sido extensivamente estudados em diversos modelos de obesidade ou mesmo de doenças crônicas não transmissíveis ${ }^{(5-7)}$. Poucos são os estudos que verificaram esses efeitos em atletas competitivos. De fato, atletas competitivos treinam exaustivamente e essa rotina de exercícios leva o organismo a um alto desgaste, principalmente se for associada à inadequada ingestão alimentar. Dessa forma, estudos avaliando a influência desses lipídios dietéticos nessa população são requeridos. Espera-se que tais pesquisas possam identificar as medidas preventivas e terapêuticas associadas a doenças cardiovasculares.

O objetivo deste estudo foi verificar os efeitos da suplementação com ácidos graxos ômega 3 (N-3) no perfil lipídico sanguíneo, no período pré-competitivo, em atletas de natação.

\section{METODOLOGIA}

Sujeitos - Quatorze atletas de natação do sexo masculino foram voluntários para este estudo. A seleção dos atletas foi realizada em um clube de natação do Rio de J aneiro, RJ , através de entrevista pessoal. Como critério de inclusão foi exigido um mínimo de dois anos de treinamento regular, não ser fumante, não ser etilista, não fazer uso de esteróides anabólicos ou similares e não possuir histórico de doenças metabólicas. Todos foram devidamente informados e assinaram declaração de consentimento esclarecido atendendo às normas da Comissão de Ética em Pesquisa do Hospital Universitário Clementino Fraga Filho da Universidade Federal do Rio de J aneiro (no 098/03 de 4-9-2003).
Análise da ingestão alimentar - Para análise quantitativa da ingestão alimentar pré-suplementação foi aplicado o registro alimentar de três dias. A análise qualitativa dos ácidos graxos ômega 3 foi avaliada por meio do questionánio de freqüência alimentar seletivo para alimentos fontes de ácido graxo ômega 3. Foi utilizado o programa de Apoio a Nutrição - Nutwin, versão 1.5/2002 para a análise de energia, carboidratos, lipídios e proteínas. A dieta habitual, apresentada na seção de resultados (tabela 1), foi ajustada para que os atletas consumissem um valor energético de 50kcal por kg de peso corporal e de proteínas, carboidratos e lipídios, respectivamente, 1,8g, $8 \mathrm{~g}$ e 1,2g em gramas por $\mathrm{kg}$ de peso corporal com base nas recomendações da Diretriz da Sociedade Brasileira de Medicina do Esporte ${ }^{(8)}$. Os atletas foram orientados para o consumo do óleo de peixe, totalizando $2,5 \mathrm{~g}$ por dia de óleo, contendo $1,8 \mathrm{~g}$ de ácidos graxos ômega-3 ( $\mathrm{n}=10$ ), ou placebo (óleo mineral) $(n=10)$. A suplementação foi realizada na forma de cápsulas, que os atletas ingeriam (cinco unidades de 500mg por dia) durante o desjejum.

Avaliação antropométrica - As medidas antropométricas foram realizadas sempre pela manhã antes da sessão de treino por profissional treinado. Todas as medidas foram feitas estando os atletas com o mínimo de roupa e descalços. As medidas foram realizadas no lado direito do corpo. Foram avaliados a espessura de dobra cutânea (peito, média axilar, tríceps, bíceps, subescapular, supra-ilíaca, abdominal, coxa e panturrilha) usando plicômetro Lange com precisão de 0,1mm; o perímetro corporal (bíceps relaxado e contraído, coxa, perna, cintura e quadril) com fita metálica flexível Cardiomed com precisão de $1 \mathrm{~mm}$; a estatura com estadiômetro Personal Sanny, com dois metros de comprimento e precisão de $1 \mathrm{~mm}$ e a massa corporal total (balança mecânica plataforma, marca Filizola, com precisão de $100 \mathrm{~g}$ ).

A partir das medidas obtidas foram calculados parâmetros como percentual de gordura corporal e massa corporal magra segundo as equações de J ackson e Pollock ${ }^{(9)}$.

Desenho do estudo - Este estudo foi randomizado, controlado por placebo. Os atletas foram divididos em dois grupos por sorteio aleatório: grupo óleo de peixe ( $\mathrm{n}=10,21,17 \pm 2$ anos de idade; $76,78 \pm 1,89 \mathrm{~kg}$ ) e grupo placebo ( $\mathrm{n}=10,20,5 \pm 1$ ano de idade; $77,13 \pm 0,86 \mathrm{~kg}$ ). Os grupos receberam a suplementação durante um período de 45 dias; coletas de sangue foram realizadas imediatamente antes (T0), aos 15 (T15), aos 30 (T30) e aos 45 (T45) dias de suplementação.

Protocolo de atividade física - Os atletas treinavam seis dias da semana em dois períodos, manhã e tarde, em média cinco horas por dia. O treinamento consistia em circuitos na água que totalizavam em média $50 \mathrm{~km} / \mathrm{semana}$. Além disso, os nadadores realizavam, em dias alternados, pelo menos uma hora de trabalho de resistência muscular.

Obtenção das amostras - As coletas de sangue venoso foram realizadas pela manhã, no próprio clube, com a colaboração de profissional especializado, mediante punção da veia antecubital com agulhas e seringas descartáveis. Após a separação do soro ou plasma, após jejum prévio de 12 horas, esse material foi encaminhado ao laboratório de Bioquímica Nutricional da UFRJ para análise de ácidos graxos plasmáticos e lipoproteínas.

Análises bioquímicas - As concentrações plasmáticas de CT (colesterol total), HDL, TG foram determinadas através de kits enzimáticos fornecidos pela Katal Biotecnológica Ind. Com. Ltda., Belo Horizonte, MG/Brasil). A mensuração de HDL-C no soro foi determinada a partir da técnica de precipitação com ácido fosfotúngstico e cloreto de magnésio. A concentração de LDL-C foi calculada usando a equação de Friedewald et al.(10). O perfil dos ácidos graxos plasmáticos foi determinado por cromatografia gasosa. A extração lipídica, saponificação e obtenção dos ácidos graxos metilados plasmáticos foram feitas de acordo com o método de Lepage e Roy(11). Os ésteres metílicos foram quantificados por cromatografia gasosa usando cromatógrafo Perkin Elmer Autosys- 
tem $X L$ equipado com detector de chama ionizável e software Turbochrom (Perkin Elmer, EUA). Os ácidos graxos metilados foram separados com coluna capilar (SP-2560 biscyanopropyl-polysiloxane, $100 \mathrm{~m} \times 0,25 \mathrm{~mm}$ de diâmetro e 0,20 mícron de espessura de filme, Supelco, Bellefonte, PA). A temperatura da corrida foi programada para iniciar a $175^{\circ} \mathrm{C}$ durante 18 minutos com subida de $1^{\circ} \mathrm{C} /$ minuto até $195^{\circ} \mathrm{C}$. A partir daí com subida de $3^{\circ} \mathrm{C}$ até alcançar $240^{\circ} \mathrm{C}$, permanecendo por cinco minutos. O tempo total da corrida foi de 58 minutos. As temperaturas do injetor e detector foram respectivamente, de $250^{\circ} \mathrm{C}$ e $270^{\circ} \mathrm{C}$. O hidrogênio foi utilizado como gás de arraste em pressão de 28 psi. A razão de split foi de 1:40. Os ésteres foram identificados por comparação com seu tempo de retenção com padrões conhecidos (Sigma, Supelco e Nuchek). Os resultados foram expressos sobre o percentual de ácidos graxos totais.

Análise estatística - Os resultados dos tempos (T0, T15, T30, T45) são apresentados nas figuras na forma de valor médio \pm erro padrão. $\mathrm{O}$ estudo das mensurações consecutivas das variáveis consideradas foi realizado por meio da análise de medidas repetidas pelo teste de Friedman para dados não paramétricos. A comparação entre grupos foi feita pelo teste $t$ de Student. Todas as conclusões estatísticas foram realizadas no nível de 5\% de significância. Os testes foram realizados pelo programa de estatística SPSS 11.0.

\section{RESULTADOS}

Perfil alimentar - A tabela 1 apresenta o perfil alimentar habitual dos nadadores. Os resultados demonstraram um desajuste na dieta dos atletas considerando a ingestão $\mathrm{g} / \mathrm{kg}$ de massa corporal dos macronutrientes.

TABELA 1

Consumo alimentar habitual de atletas nadadores pré-suplementação com óleo de peixe

Registro alimentar de três dias

Energia (kcal)

$\mathrm{kcal} / \mathrm{kg}$ de $\mathrm{PC}$

Proteínas (\%)

Proteínas (g)

Proteínas (g/kg de PC)

Carboidratos (\%)

Carboidratos (g)

Carboidratos ( $\mathrm{g} / \mathrm{kg}$ de $\mathrm{PC}$ )

Lipídios (\%)

Lipídios (g)

Lipídios ( $\mathrm{g} / \mathrm{kg}$ de PC)
A análise do questionário de freqüência de consumo mostrou que os atletas não consumiam regularmente fontes alimentares de ômega 3 e que o consumo de peixes em $85 \%$ da amostra era inferior ou igual a uma vez na semana (figura 1 ).

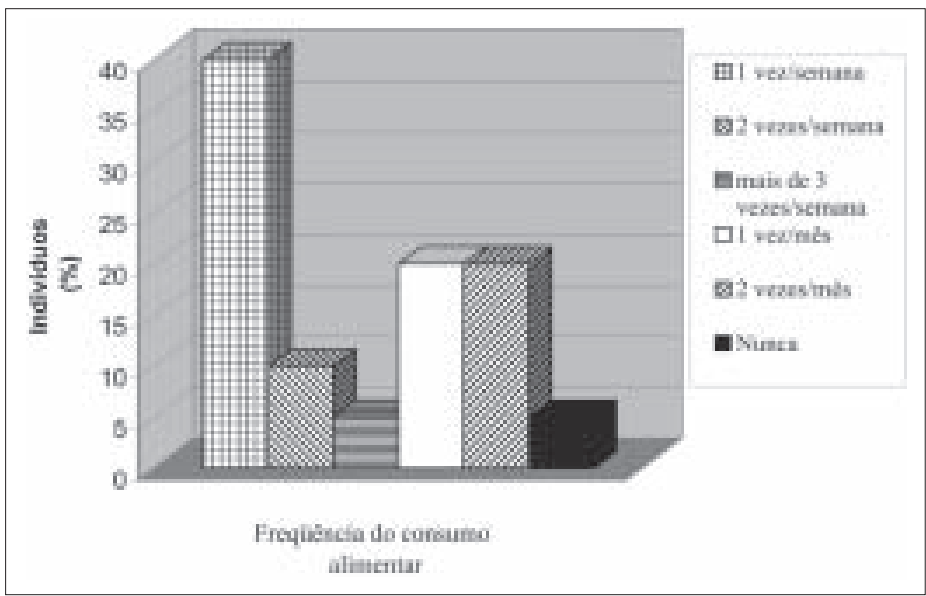

Figura 1 - Distribuição percentual do consumo de peixes segundo questionário de freqüência alimentar, em atletas nadadores

Perfil antropométrico - As características antropométricas dos atletas recrutados neste estudo encontram-se na tabela 2 . Os resultados são apresentados como média \pm erro padrão. A idade média foi de $21,17 \pm 2$ anos. A análise estatística dos dados indicou valores menores com significância estatística $(P=0,042)$ para o somatório das dobras cutâneas após o período de suplementação com óleo de peixe. Os demais parâmetros analisados não apresentaram qualquer diferença significativa.

Parâmetros bioquímicos - Para análise dos dados bioquímicos, utilizamos " $n$ " amostral de seis sujeitos do grupo suplementado e oito do grupo placebo, considerando que seis indivíduos (quatro do grupo suplementado e dois do grupo placebo) não compareceram às últimas coletas de sangue (30 e 45 dias do estudo).

Concentração dos ácidos graxos no plasma - O perfil de ácidos graxos plasmáticos encontra-se descrito na tabela 3, sendo os valores apresentados como média \pm erro padrão da média (EPM). A análise plasmática dos ácidos graxos saturados, para ambos os grupos, mostra inicialmente diminuição nos valores percentuais seguido de aumento até o final do estudo, sendo esta oscilação diferente estatisticamente apenas para o grupo óleo de peixe $(P=0,047)$. No grupo suplementado os valores percentuais dos ácidos graxos esteárico (18:0) e palmítico (16:0) foram significativamente maiores que os valores do grupo placebo aos 30 dias do estudo.

\begin{tabular}{lccccc}
\hline \multicolumn{3}{c}{ TABELA 2 } \\
Variáveis antropométricas dos atletas nadadores \\
antes e após a suplementação com óleo de peixe
\end{tabular}

Valores são média \pm EPM. $\Sigma$ - somatório; T0 - coleta realizada antes da suplementação; T45 - coleta realizada 45 dias após início da suplementação. Placebo, suplemento alimentar isento de ômega 3. Grupo óleo de peixe - indivíduos que receberam cinco cápsulas de óleo de peixe $(n=8)$ contendo $950 \mathrm{mg}$ de ácido eicosapentaenóico e 500mg de ácido docosapentaenóico por dia. * $p<0,05$ indica diferença significativa com relação ao TO. 
Os valores percentuais dos ácidos graxos monoinsaturados no plasma diminuem no grupo suplementado com óleo de peixe ao longo do período do estudo. Aos 30 dias do estudo, o grupo suplementado com óleo de peixe apresentou diminuição significativa nos valores percentuais dos ácidos graxos palmitoléico (16:1) e oléico (18:1 n-9 cis) em relação aos valores do grupo placebo. Já aos 45 dias essa diminuição foi significativa apenas com o ácido oléico (tabela 3).

A análise de ácidos graxos trans (18:1 n-9) no plasma dos atletas não apresentou diferenças significativas entre os grupos.

Ainda na tabela 3, observa-se que os valores percentuais dos ácidos graxos poliinsaturados N-6 linoléico (18:2) e araquidônico (20:4) mostraram diminuição ao longo do período de suplementação com óleo de peixe, ainda que diferença significativa só foi observada para o ácido araquidônico no grupo suplementado $(p=$ 0,008 ) ao longo do estudo. No grupo placebo, os valores encontrados para o ácido linoléico aumentaram entre o 150 e o 30 ㅇa dia do estudo, sendo que aos 45 dias os valores se aproximaram dos valores iniciais, sendo esta oscilação significativa $(p=0,013)$. Os resultados obtidos para o ácido araquidônico no grupo placebo mostram valores aumentados, com significância estatística, ao longo do estudo ( $p=0,048$ ). Quando se comparam os valores dos AGPI N-6 entre os grupos placebo e óleo de peixe, observa-se que a percentagem de linoléico foi menor no grupo óleo de peixe aos 15 e 30 dias do estudo. Já a percentagem de araquidônico apresentou valores significativamente mais baixos no grupo óleo de peixe aos 30 e 45 dias.

Observa-se aumento significativo, ao longo do estudo, dos ácidos graxos poliinsaturados N-3 EPA $(p=0,002)$ e DHA $(p=0,01)$ no grupo suplementado com óleo de peixe. Os valores percentuais do ácido graxo 18:3 N-3 (ácido linolênico) se mantiveram estáveis no grupo placebo ao longo do estudo enquanto no grupo óleo de peixe os valores mostraram oscilação, inicialmente elevando-se aos 15 dias seguido de diminuição aos 30 dias e novamente aumentando aos 45 dias, com diferença significativa estatística na análise ao longo do tempo $(p=0,018)$.

Comparativamente ao grupo placebo, os valores percentuais de EPA e DHA foram maiores no grupo suplementado com óleo

TABELA 3

Perfil plasmático de ácidos graxos de atletas nadadores antes e após a suplementação com óleo de peixe

\begin{tabular}{|c|c|c|c|c|c|c|c|c|c|c|}
\hline \multirow[t]{2}{*}{ Ácidos graxos } & \multicolumn{2}{|c|}{ Antes (zero) } & \multicolumn{2}{|c|}{15 dias } & \multicolumn{2}{|c|}{30 dias } & \multicolumn{2}{|c|}{45 dias } & \multicolumn{2}{|c|}{$P$ valor $\phi$} \\
\hline & Placebo & $\begin{array}{c}\text { Óleo } \\
\text { de peixe }\end{array}$ & Placebo & $\begin{array}{c}\text { Óleo } \\
\text { de peixe }\end{array}$ & Placebo & $\begin{array}{c}\text { Óleo } \\
\text { de peixe }\end{array}$ & Placebo & $\begin{array}{c}\text { Óleo } \\
\text { de peixe }\end{array}$ & Placebo & $\begin{array}{c}\text { Óleo } \\
\text { de peixe }\end{array}$ \\
\hline Saturados & $\mathbf{N}=\mathbf{6}$ & $\mathbf{N}=\mathbf{8}$ & $\mathbf{N}=6$ & $\mathbf{N}=\mathbf{8}$ & $\mathbf{N}=6$ & $\mathbf{N}=\mathbf{8}$ & $\mathbf{N}=\mathbf{6}$ & $\mathbf{N}=\mathbf{8}$ & & \\
\hline $\begin{array}{l}\text { Esteárico (C18:0) } \\
\text { Palmítico (C16:0) } \\
\text { Total }\end{array}$ & $\begin{array}{l}9,101 \pm 0,56 \\
23,57 \pm 1,19 \\
38,47 \pm 1,05\end{array}$ & $\begin{array}{r}9,57 \pm 0,65 \\
25,21 \pm 0,85 \\
40,98 \pm 1,08\end{array}$ & $\begin{array}{r}8,38 \pm 0,51 \\
26,94 \pm 0,53 \\
37,94 \pm 1,63\end{array}$ & $\begin{array}{r}8,59 \pm 0,69 \\
24,92 \pm 0,83 \\
39,11 \pm 0,66\end{array}$ & $\begin{array}{r}7,45 \pm 0,39 \\
25,77 \pm 0,82 \\
36,41 \pm 0,40\end{array}$ & $\begin{array}{l}10,60 \pm 0,77 * * \\
28,24 \pm 0,32^{* *} \\
43,76 \pm 0,86^{* * *}\end{array}$ & $\begin{array}{l}10,73 \pm 1,58 \\
25,81 \pm 0,58 \\
44,76 \pm 2,18\end{array}$ & $\begin{array}{l}10,94 \pm 1,43 \\
25,94 \pm 1,09 \\
45,22 \pm 1,65\end{array}$ & $\begin{array}{l}0,06 \mathrm{~ns} \\
0,06 \mathrm{~ns} \\
0,172 \mathrm{~ns}\end{array}$ & $\begin{array}{l}0,016 \mathrm{~s} \\
0,041 \mathrm{~s} \\
0,047 \mathrm{~s}\end{array}$ \\
\hline \multicolumn{11}{|l|}{ Monoinsaturados } \\
\hline $\begin{array}{l}\text { Palmitoléico (C16:1) } \\
\text { Oleico (C18:1 n9cis) } \\
\text { Total }\end{array}$ & $\begin{array}{r}1,32 \pm 0,20 \\
20,24 \pm 2,07 \\
21,88 \pm 2,23\end{array}$ & $\begin{array}{r}1,14 \pm 0,14 \\
19,11 \pm 1,69 \\
20,55 \pm 1,68\end{array}$ & $\begin{array}{r}1,23 \pm 0,27 \\
14,13 \pm 1,12 \\
16,03 \pm 1,42\end{array}$ & $\begin{array}{r}1,04 \pm 0,17 \\
12,023 \pm 0,52 \\
14,44 \pm 0,78\end{array}$ & $\begin{array}{r}0,95 \pm 0,08 \\
13,04 \pm 0,59 \\
14,52 \pm 0,70\end{array}$ & $\begin{array}{r}0,55 \pm 0,12^{* *} \\
10,74 \pm 0,39^{* *} \\
11,58 \pm 0,51^{* *}\end{array}$ & $\begin{array}{l}0,911 \pm 0,03 \\
12,81 \pm 0,72 \\
14,47 \pm 0,66\end{array}$ & $\begin{array}{c}0,96 \pm 0,19 \\
9,89 \pm 0,95 * \\
11,30 \pm 1,09\end{array}$ & $\begin{array}{l}0,392 \mathrm{~ns} \\
0,102 \mathrm{~ns} \\
0,060 \mathrm{~ns}\end{array}$ & $\begin{array}{l}0,041 \mathrm{~s} \\
0,001 \mathrm{~s} \\
0,001 \mathrm{~s}\end{array}$ \\
\hline Ácidos graxos Trans & $1,1 \pm 0,15$ & $1,38 \pm 0,09$ & $1,15 \pm 0,25$ & $1,44 \pm 0,25$ & $1,08 \pm 0,03$ & $1,10 \pm 0,01$ & $1,33 \pm 0,15$ & $1,79 \pm 0,27$ & $0,706 \mathrm{~ns}$ & $0,199 \mathrm{~ns}$ \\
\hline
\end{tabular}

\section{Poliinsaturados}

N 6

Linoléico (C18:2) Araquidônico (C20:4)

Total

$25,31 \pm 1,88 \quad 24,03 \pm 1,44$

$4,69 \pm 0,63 \quad 4,04 \pm 0,41$

$29,32 \pm 1,59 \quad 23,05 \pm 2,03 *$

$35,59 \pm 2,15 \quad 27,95 \pm 2,46$

$29,85 \pm 1,15 \quad 23,95 \pm 1,28 * *$

$5,08 \pm 0,26 * \quad 3,77 \pm 0,10 * * *$

$23,26 \pm 1,40 \quad 22,56 \pm 1,42$

$35,40 \pm 1,79 \quad 29,1 \pm 1,48$

$5,63 \pm 0,29 \quad 3,07 \pm 0,21 * *$

$0,013 \mathrm{~s} \quad 0,256 \mathrm{~ns}$

$0,048 \mathrm{~s} \quad 0,008 \mathrm{~s}$

0,041 s $\quad 0,510$ ns

N 3

Linolênico (C18:3)

EPA (C20:5)

DHA (C22:6)

Total

$\begin{array}{cc}0,217 \pm 0,3 & 0,24 \pm 0,02 \\ 0,47 \pm 0,17 & 0,50 \pm 0,19 \\ 1,76 \pm 0,19 & 2,70 \pm 0,45 \\ 3,17 \pm 0,42 & 4,39 \pm 0,55\end{array}$

$0,39 \pm 0,07 \quad 0,597 \pm 0,11$

$0,26 \pm 0,02 \quad 1,09 \pm 0,27$

$0,48 \pm 0,07 \quad 0,17 \pm 0,04 * *$

$29,92 \pm 1,7927,96 \pm 2,07$

0,133 ns $0,018 \mathrm{~s}$

$\begin{array}{llll}1,29 \pm 0,32 & 3,84 \pm 0,67 * * & 2,99 \pm 0,40 & 2,55 \pm 0,38\end{array}$

$0,34 \pm 0,07 \quad 0,48 \pm 0,12$

$0,23 \pm 0,04 \quad 1,48 \pm 0,51$

0,362 ns $0,002 \mathrm{~s}$

$2,39 \pm 0,41 \quad 5,69 \pm 0,90 \quad 4,03 \pm 0,54 \quad 3,8 \pm 0,53$

$1,87 \pm 0,18 \quad 4,07 \pm 0,72 *$

$0,092 \mathrm{~ns} \quad 0,01 \mathrm{~s}$

Total (N6 + N3)

$33,31 \pm 2,8833,37 \pm 1,79$

$37,98 \pm 2,49 \quad 33,63 \pm 4,64$

$39,45 \pm 2,5 \quad 32,9 \pm 2,37$

$32,84 \pm 2,46 \quad 33,69 \pm 1,72$

$0,069 \mathrm{~ns} \quad 0,934 \mathrm{~ns}$

Relação N6/ N3

$10,21 \pm 1,40 \quad 9,58 \pm 1,96 \quad 15,00 \pm 2,50 \quad 6,13 \pm 1,20$

$10,22 \pm 2,39 \quad 6,13 \pm 0,36 *$

$11,00 \pm 1,16 \quad 7,17 \pm 1,51$

$0,095 \mathrm{~ns} \quad 0,419 \mathrm{~ns}$

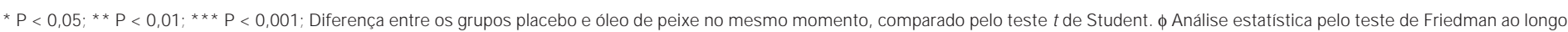
do tempo em cada grupo do estudo. S: significativo $(P<0,05)$ e NS não significativo $(P>0,05)$.

TABELA 4

Perfil plasmático de lipoproteínas de atletas nadadores antes e após a suplementação com óleo de peixe

\begin{tabular}{|c|c|c|c|c|c|c|c|c|c|c|}
\hline \multirow[t]{2}{*}{ Lipoproteínas } & \multicolumn{2}{|c|}{ Antes (zero) } & \multicolumn{2}{|c|}{15 dias } & \multicolumn{2}{|c|}{30 dias } & \multicolumn{2}{|c|}{45 dias } & \multicolumn{2}{|c|}{ P valor $\phi$} \\
\hline & Placebo & $\begin{array}{c}\text { Óleo } \\
\text { de peixe }\end{array}$ & Placebo & $\begin{array}{c}\text { Óleo } \\
\text { de peixe }\end{array}$ & Placebo & $\begin{array}{c}\text { Óleo } \\
\text { de peixe }\end{array}$ & Placebo & $\begin{array}{c}\text { Óleo } \\
\text { de peixe }\end{array}$ & Placebo & $\begin{array}{c}\text { Óleo } \\
\text { de peixe }\end{array}$ \\
\hline $\mathrm{mg} / \mathrm{dL}$ & $N=6$ & $\mathbf{N}=\mathbf{8}$ & $N=6$ & $\mathbf{N}=\mathbf{8}$ & $\mathbf{N}=6$ & $\mathbf{N}=\mathbf{8}$ & $N=6$ & $\mathbf{N}=\mathbf{8}$ & & \\
\hline HDL & $46,94 \pm 7,14$ & $42,49 \pm 4,62$ & $43,08 \pm 3,73$ & $38,33 \pm 2,68$ & $46,03 \pm 3,76$ & $40,93 \pm 0,96$ & $46,69 \pm 1,93$ & $41,59 \pm 2,58$ & $0,158 \mathrm{~ns}$ & $0,175 \mathrm{~ns}$ \\
\hline VLDL & $17,66 \pm 1,77$ & $17,78 \pm 1,60$ & $20,52 \pm 2,71$ & $17,22 \pm 1,86$ & $19,87 \pm 1,97$ & $13,37 \pm 1,75^{*}$ & $20,17 \pm 2,19$ & $13,99 \pm 1,34 *$ & $0,334 \mathrm{~ns}$ & $0,027 \mathrm{~s}$ \\
\hline LDL & $117,5 \pm 16,6$ & $111,9 \pm 13,7$ & $95,29 \pm 10,62$ & $78,88 \pm 5,4$ & $93,94 \pm 8,6$ & $76,92 \pm 4,38$ & $105,2 \pm 9,27$ & $74,2 \pm 2,49 * *$ & $0,706 \mathrm{~ns}$ & $0,062 \mathrm{~ns}$ \\
\hline $\mathrm{CHOL}$ & $182,1 \pm 15,5$ & $172,2 \pm 6,37$ & $158,9 \pm 11,86$ & $134,4 \pm 4,34 *$ & $163,5 \pm 6,46$ & $131,2 \pm 5,45^{* *}$ & $168,7 \pm 8,45$ & $129,8 \pm 1,39 * * *$ & $0,896 \mathrm{~ns}$ & $0,034 \mathrm{~s}$ \\
\hline TG & $88,29 \pm 8,84$ & $88,92 \pm 8,02$ & $102,59 \pm 13,5$ & $86,08 \pm 9,3$ & $99,3 \pm 9,86$ & $66,83 \pm 8,77^{*}$ & $100,8 \pm 10,9$ & $69,9 \pm 6,71^{*}$ & $0,334 \mathrm{~ns}$ & $0,027 \mathrm{~s}$ \\
\hline CHOL/HDL & $4,32 \pm 0,64$ & $4,27 \pm 0,48$ & $3,73 \pm 0,22$ & $3,65 \pm 0,29$ & $3,47 \pm 0,36$ & $3,23 \pm 0,19$ & $3,38 \pm 0,19$ & $3,21 \pm 0,20$ & $0,308 \mathrm{~ns}$ & $0,021 \mathrm{~s}$ \\
\hline LDL/HDL & $2,90 \pm 0,56$ & $2,81 \pm 0,41$ & $2,23 \pm 0,22$ & $2,16 \pm 0,24$ & $2,06 \pm 0,35$ & $1,89 \pm 0,14$ & $2,1 \pm 0,18$ & $1,85 \pm 0,16$ & $0,572 \mathrm{~ns}$ & $0,016 \mathrm{~s}$ \\
\hline
\end{tabular}

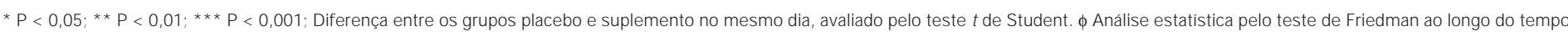
em cada grupo do estudo. S: significativo $(P<0,05)$ e NS não significativo $(P>0,05)$. 
de peixe durante todo o período do estudo, apresentando diferença significativa aos 30 dias para o EPA $(p=0,0009)$ e aos 15 e 45 dias para o DHA, respectivamente, $p=0,0093$ e 0,0019.

No que diz respeito à relação AGPI N-6/N-3 plasmática observa-se na tabela 3 que o grupo suplementado com óleo de peixe apresentou esta relação mais baixa comparativamente ao do grupo placebo; entretanto, com significância estatística apenas aos 30 dias do estudo $(P<0,05)$.

Perfil lipoprotéico - O perfil das lipoproteínas plasmáticas encontra-se descrito na tabela 4. Em relação às concentrações plasmáticas de HDL, não se observa qualquer diferença significativa, nem ao longo do tempo, nem em função da suplementação com óleo de peixe. Os valores plasmáticos encontrados para o LDL evidenciaram tendência de diminuição ao longo do tempo $(p=$ 0,06 ) para o grupo suplementado, o mesmo não sendo observado para o grupo controle. Quando se comparam, individualmente, os tempos entre os grupos, a diferença alcança nível de significância com valores de $p<0,01(p=0,0031)$ aos 45 dias entre o grupo placebo e o suplementado.

Ao longo do tempo do estudo observa-se diminuição significativa nas concentrações plasmáticas de VLDL $(p=0,027)$, TG ( $p=$ 0,027 ) e colesterol total $(p=0,034)$ no grupo suplementado com óleo de peixe. Quando se comparam individualmente os tempos entre os grupos, essas diferenças apresentam significância estatística a partir do 350 dia.

A relação $\mathrm{CHOL} / \mathrm{HDL}$ e LDL/HDL diminui significativamente ao longo do tempo do estudo no grupo suplementado com óleo de peixe.

\section{DISCUSSÃO}

Os ácidos graxos poliinsaturados ômega-3 (AGPI N-3) vem atraindo a atenção de inúmeros pesquisadores pelo seu papel em estimular o metabolismo lipídico e tumover de lipoproteínas(12). Além disso, a maior ingestão de AGPI N-3 vem acompanhada de aumento da concentração desses ácidos graxos no plasma, o que, por sua vez, pode ser importante para a dinâmica imunológica do indivíduo ${ }^{(13)}$ e na proteção contra eventos cardiovasculares ${ }^{(14)}$.

Dentro desse contexto, nossa proposta de trabalho foi investigar em atletas de natação a ação desses ácidos graxos nos mediadores bioquímicos associados ao metabolismo lipídico, utilizando como modelo de intervenção nutricional a suplementação de ácidos graxos ômega 3 com cápsulas de óleo de peixe administradas nos 45 dias que antecederam a data da competição.

Após a suplementação com cápsulas de óleo de peixe $(2,5 \mathrm{~g} /$ dia) foi observado aumento nas concentrações plasmáticas dos ácidos graxos N-3 eicosapentaenóico (EPA) e docosapentaenóico (DHA). Antagonicamente, observa-se redução nas concentrações de ácidos graxos poliinsaturados $\mathrm{N}-6$, principalmente, do ácido araquidônico (AA) e do ácido linoléico, mostrando assim que o modelo de suplementação proposto no estudo refletiu a ingestão dietética, sendo capaz de proporcionar níveis de ácidos graxos ômega 3 no sangue compatíveis para verificar alterações fisiológicas e bioquímicas no organismo.

De maneira similar, Foulon et al.(15) também evidenciaram, após suplementação de $\mathrm{N}-3$ (1,8g/dia) em indivíduos saudáveis por três semanas, redução dos níveis plasmáticos de ácidos graxos poliinsaturados $\mathrm{N}-6$ e aumento nas concentrações plasmáticas de N-3; no entanto, não verificaram alterações significativas nas concentrações sanguíneas de colesterol total, LDL e TG, contrariamente aos nossos resultados, que evidenciaram uma redução nesses marcadores. Estas diferenças poderiam ser, em parte, explicadas pelo tempo de suplementação adotado em ambos os estudos, uma vez que no nosso estudo suplementamos por período mais longo (seis semanas).

No presente estudo o percentual dos AG saturados plasmáticos aumentou no grupo suplementado com óleo de peixe ao lon- go do tempo (tabela 3). Quando comparamos ambos os grupos estudados, observa-se que o aumento foi significativo no grupo suplementado $(p<0,05 ; p=0,047)$ em relação ao placebo. Comportamento inverso foi encontrado para os ácidos graxos monoinsaturados, cujos valores ao longo do tempo foram significativamente menores no grupo óleo de peixe, bem como em relação ao placebo aos 30 dias. Esses resultados indicam que, em exercícios de grande intensidade, provavelmente, os ácidos graxos monoinsaturados são preferencialmente captados pelos tecidos, principalmente o muscular, tanto para repor seus estoques de TG intramuscular (TGIM) como para prover energia. Alguns pesquisadores sugerem que o treinamento não aumenta a oxidação dos AG provenientes do plasma, mas provavelmente aumenta o estoque de TGIM(16). Outra possível explicação para essas alterações observadas no grupo óleo de peixe seja decorrente da menor dessaturação dos ácidos graxos esteárico (C18:0 N-9) e palmítico (C16:0 $\mathrm{N}-7)$ resultando na diminuição da síntese de oléico (C18:1 n-9) e palmitoléico (C16:1 N-7) respectivamente e, por causa disso, menores proporções desses ácidos graxos no plasma e aumentados níveis dos seus precursores.

As enzimas de dessaturação e alongamento podem agir não só nas séries de ácidos graxos poliinsaturados N-3 e N-6, mas também nos ácidos graxos N-9 e N-7, ocorrendo inclusive competição entre os substratos. Além disso, as velocidades de dessaturação e alongamento diferem entre as séries, decrescendo na ordem $\mathrm{N}-3>\mathrm{N}-6>\mathrm{N}-9>\mathrm{N}-7$. Assim, maiores concentrações de ácidos graxos da série $\mathrm{N}-3$ na dieta podem inibir a dessaturação de outras séries de ácidos graxos como N-9 e N-7(17).

Os TG não são essencialmente componentes estruturais da lesão aterosclerótica; no entanto, elevadas concentrações de TG no plasma podem exibir um efeito adverso por promover a produção de quilomícrons remanescentes aterogênicos ${ }^{(18)}$. Além disso, altas concentrações de TG podem induzir o aumento do teor de ésteres de colesterol na HDL pela ação da enzima CETP (cholesterol ester transfer protein), interferindo no efeito benéfico que o HDL teria no metabolismo do colestero|(18). Conseqüentemente, terapias que melhoram o metabolismo lipoprotéico poderiam reduzir a incidência de deslipidemias, as quais estão associadas ao risco para doença arterial coronariana.

Os resultados do presente estudo demonstraram que a suplementação de ácidos graxos ômega 3 é importante modulador do metabolismo das lipoproteínas, suportando a hipótese de que o consumo de AGPI N-3 influenciaria favoravelmente toda a dinâmica das lipoproteínas no organismo em atletas competitivos. Embora as concentrações no soro de HDL-C não tenham alcançado significância estatística, provavelmente devido ao pequeno tamanho amostral, no entanto, quando se avaliam a relação LDL:HDL e a relação CT:HDL-C, observa-se que houve uma redução significativa (tabela 4).

Estudo em humanos mostram que os AGPI N-3 exercem consistente efeito hipotrigliceridêmico. Em trabalho de revisão realizado por Harris(19), evidenciou-se que 72 estudos controlados por placebo foram suplementados de 1 a $7 \mathrm{~g}$ diárias de EPA e DHA por, no mínimo, duas semanas e as concentrações plasmáticas de TG foram reduzidas de 25 a 30\% em geral. A duração do período de suplementação é também um fator importante, pois baixas doses de AGPI N-3 suplementadas por um longo período de tempo teriam um efeito hipotrigliceridêmico semelhante ao de suplementações por períodos mais curtos e com altas doses. A suplementação de $1 \mathrm{~g}$ de AGPI N-3 por 12 semanas reduziu significativamente as concentrações de TG de jejum em $21 \%{ }^{(18)}$. A importância da duração da suplementação é confirmada pelos resultados de outros estudos. Schmidt et al.(20) demonstraram que o aporte de $4 \mathrm{~g}$ de AGPI N-3 por nove meses também reduziu os valores de TG no plasma. Os trabalhos conduzidos por Saynor e Gilliott ${ }^{(21)}$ mostram uma redução contínua quando a suplementação se dá por períodos maiores que quatro anos. No presente estudo foi verificado 
que doses de 1,8g por dia por período de seis semanas já são eficazes em promover uma redução dos triglicérides plasmáticos. De qualquer forma, estudos relacionando a suplementação com ácidos graxos ômega 3 em atletas nadadores e o perfil lipoprotéico são escassos, dificultando qualquer análise comparativa entre os nossos resultados e os da literatura.

Evidências mostram que os ácidos graxos N-3 reduzem as concentrações de TG através da redução na produção endógena de VLDL (22). Estudos conduzidos com animais mostram que os AGPI N-3 inibem a síntese de TG (pela inibição da enzima 1,2 diacilgliceroltransferase) e a síntese e secreção da VLDL(23-24). Todavia, os mecanismos bioquímicos precisos envolvidos nesse processo ainda precisam ser mais claramente elucidados. Outra possível explicação para esse efeito hipotrigliceridêmico do ômega 3 seria aumento na degradação dos quilomícrons decorrente de alteração no metabolismo da enzima lipase lipoprotéica em termos de concentração enzimática, atividade ou afinidade.

$\mathrm{O}$ efeito hipocolesterolêmico dos ácidos graxos N-3 pode ser atribuído à redução da taxa de formação da LDL, pois tanto o EPA quanto o DHA seriam eficazes em estimular a atividade do seu receptor no tecido hepático, contribuindo ainda mais para a redução das concentrações de LDL ${ }^{(25)}$. Este efeito ainda pode ser atribuído à supressão da atividade da enzima HMG-CoA redutase no tecido hepático(26).

Em conclusão, a suplementação de óleo de peixe, rico em ácidos graxos ômega 3 é importante para a manutenção do perfil de

\section{REFERÊNCIAS}

1. Schmidt EB, Skou HA, Christensen J H, Dyerberg J . N-3 fatty acids from fish and coronary artery disease: implications for public health. Public Health Nutr. 2000; 3(1):91-8.

2. Simopoulos AP. The importance of ratio omega-6/omega-3 essential fatty acids. Biomed Pharmacother. 2002;56:365-79.

3. Diniz YS, Cicogna AC, Padovani CR, Santana LS, Faine LA, Novelli EL. Diets rich in saturated and polyunsaturated fatty acids: metabolic shifting and cardiac health. Nutrition. 2004;20(2):230-4.

4. Whelton SP, He J, Whelton PK, M untner P. Meta-analysis of observational studies on fish intake and coronary heart disease. Am J Cardiol. 2004;93(9):1119-23.

5. Hu FB, Manson J E, Willet WC. Types of dietary fat and risk of coronary heart disease: a critical review. J Am Coll Nutr. 2001;20(1):5-19.

6. Toborek M, Lee YW, Garrido R, Kaiser S, Henning B. Unsaturated fatty acids selectively induce an inflammatory environment in human endothelial cell. Am J Clin Nutr. 2002;75:119-25.

7. Mabile L, Piolot A, Boulet L, Fortin LJ, Doyle N, Rodriguez C, et al. Moderate intake of N-3 fatty acids is associated with stable erythrocyte resistence to oxidative stress in hypertriglyceridemic subjects. Am J Clin Nutr. 2001;74:449-56.

8. Carvalho T, et al. Diretriz da Sociedade Brasileira de Medicina do Esporte. Rev Bras Med Esporte. 2003;9(2):43-56.

9. J ackson A, Pollock M. Generalized equations for predicting body density of men. BrJ Nutr. 1978;40:497-504.

10. Friedewald WT, Levy RI, Fredrickson DS. Estimation of the concentration of lowdensity lipoprotein cholesterol in plasma, without use of the preparative ultracentrifuge. Clin Chem. 1972;18:499-502.

11. Lepage G, Roy C. Direct transesterification of all classes of lipid in a one step reaction. J Lipid Res. 1986;27:114-20.

12. Gill J M, Caslake MJ , McAllister C, Tsofliou F, Ferrell WR, Packard CJ, et al. Effects of short-term detraining on postprandial metabolism, endothelial function, and inflammation in endurance-trained men: dissociation between changes in triglyceride metabolism and endothelial function. J Clin Endocrinol Metab. 2003;(9):4328-35.

13. König D, Berg AC, Weinstock C, Keul J Northoff H. Essential fatty acids, immune function and exercise. Exerc Immunol Rev. 1997;3:1-31. lipoproteínas adequado, além de promover um estímulo para a oxidação de ácidos graxos. Ainda que efeitos benéficos no perfil de lipoproteínas sejam apontados na literatura após suplementação com N-3, há uma escassez de trabalhos conduzidos com atletas de elite no Brasil. Este estudo, em atletas nadadores, demonstra o efeito da suplementação de AGPI N-3 em elevar as concentrações plasmáticas de ácidos graxos EPA e DHA. Assim, o modelo descrito para a suplementação de cápsulas de óleo de peixe em atletas competitivos poderia ser importante terapêutica para a manutenção do perfil lipoprotéico adequado nesta população. Ainda que, para que se conheça o real potencial da utilização do ácidos graxos ômega 3, são necessários estudos posteriores com outros atletas nadadores e com outras modalidades esportivas, para que se confirmem essas observações. Como proposta futura, seria também interessante investigar o efeito dose-resposta atribuídos à suplementação dietética de ácidos graxos ômega 3 em atletas.

\section{AGRADECIMENTOS}

Gostaríamos de agradecer à empresa Roche, São Paulo, Brasil, pelo fornecimento do óleo de peixe para a realização deste estudo.

Apoio: Conselho Nacional de Desenvolvimento Científico e Tecnológico (CNPq).

Todos os autores declararam não haver qualquer potencial conflito de interesses referente a este artigo.

14. Olchawa B, Kingwell BA, Hoang A, Schneider L, Miyazaki O, Nestel P, et al. Physical fitness and reverse cholesterol transport. Arterioscler Thromb Vasc Biol. 2004; 24(6):1087-91.

15. Foulon T, Richard MJ , Payen N, Bourrain J L, Beani J C, Laporte F, et al. Effects of fish oil fatty acids on plasma lipids and lipoproteins and oxidant-antioxidant imbalance in healthy subjects. Scand J Clin Lab Invest. 1998;59(4):239-48.

16. Curi R, Lagranha CJ , Hirabara SM, Folador A, Tchaikovski J r O, Fernandes LC, et al. A limiting step for fatty acids oxidation during aerobic exercise: the Krebs cycle. R Bras Cienc e Mov. 2003;11(2):87-94.

17. Calder FC. N-3 fatty acids and cardiovascular disease: evidence explained and mechanisms explored. Clinical Science. 2004;107:1-11.

18. Roche HM, Gibney MJ . Long-chain n-3 polyunsaturated fatty acids and triacylglycerol metabolism in postprandial state. Lipids. 1999;34:S259-65.

19. Harris WS. N3 fatty acids and lipoproteins: comparison of results from human and animal studies. Lipids. 1996;31:243-52.

20. Schmidt EB, Lervang HH, Varming K, Madsen P, Dyerberg J . Long-term supplementation with N-3 fatty acids, II: Effect on blood lipids, haemostasis and blood pressure. Scand J Clin Lab Invest. 1992;52(3):221-8.

21. Saynor R, Gilliott T. Changes in blood lipids and fibrinogen with a note on safety in a long-term study on the effects of N-3 fatty acids in subjects receiving fish oil supplements and followed for seven years. Lipids. 1992;27:533-8.

22. Baker PW, Gibbons GF. Effect of dietary fish oil on the sensitivity of hepatic lipid metabolism to regulation by insulin. J Lipid Res. 2000;41(5):719-26.

23. Rustan AC, Nossen J O, Christiansen EN, Drevon CA. Eicosapentaenoic acid reduces hepatic synthesis and secretion of triacylglycerol by decreasing the activity of acyl-coenzyme A:1,2 diacylglycerol acyltransferase. J Lipid Res. 1988;29: 1417-26.

24. Rustan AC, Drevon CA. Eicosapentaenoic acid inhibits hepatic production of very low density lipoprotein. J Intern Med Suppl. 1989;731:31-8.

25. Spady DK. Regulatory effects of individual $n-6$ and $n-3$ polyunsaturated fatty acids on LDL transport in the rat. J Lipid Res. 1993;34(8):1337-46.

26. Du C, Sato A, Watanabe S, Wu CZ, Ikemoto A, Ando K, et al. Cholesterol synthesis in mice is suppressed but lipofuscin formation is not affected by long-term feeding of n-3 fatty acid-enriched oils compared with lard and n-6 fatty acid-enriched oils. Biol Pharm Bull. 2003;26(6):766-70. 